\title{
The effect of lipid lowering agents on improving depression-like behavior in metabolic syndrome mice
}

\author{
Ching-Hua Yeh ${ }^{1}$, Liang-Po Hsieh ${ }^{1,2}$
}

${ }^{I}$ Department of Medicinal Botanicals and Health Applications, Da-Yeh University, Changhua, Taiwan, ${ }^{2}$ Department of Neurology, Cheng Ching General Hospital, Taichung, Taiwan

With the changing lifestyle, high-calorie diet and lack of exercise makes the number of metabolic syndrome patients gradually increased. Depression is linked to metabolic syndrome and its components, including dyslipidemia, and central obesity. Metabolic syndrome would be a significant predictor of depression. In this study, we evaluated whether fenofibrate(FF) or statin, could improve depression-like behavior in metabolic syndrome mice. The metabolic syndrome mice were induced by high-fat-diet. The depression-like behaviors in metabolic syndrome mice were analyzed by tail suspension test, open field test and sucrose preference test. After 4-week treatment of fenofibrtae and Statin, the depression-like behavior in mice were analyzed and then sacrificed. The expressions of inflammatory cytokines in the brain of mice were detected by RT-PCR. The activation of macrophage and microglia were analyzed using IHC stain. The results showed that body weight, TG, TC in serum and adipose tissue weight of mice were increased after high-fat-diet fed. That confirmed metabolic syndrome was induced successfully in the mice. In behavior analysis, fenofibrate decreased immobility times of tail suspension, increased number of line crossing of open field test, and increase sucrose intake of sucrose preference test indicating that fenofibrate may improve depression-like behavior. The data showed that fenofibrate could improve depression level in metabolic syndrome mice. 